International Journal of Modern Physics A

(C) World Scientific Publishing Company

\title{
BLACK HOLE PRODUCTION AT THE LARGE HADRON COLLIDER
}

\author{
DOUGLAS M. GINGRICH* \\ Centre for Particle Physics, Department of Physics, University of Alberta, \\ Edmonton, AB T6G 2G7, Canada \\ gingrich@ualberta.ca
}

Received November 2, 2018

\begin{abstract}
Black hole production at the Large Hadron Collider (LHC) is an interesting consequence of $\mathrm{TeV}$-scale gravity models. The predicted values, or lower limits, for the fundamental Planck scale and number of extra dimensions will depend directly on the accuracy of the black hole production cross-section. We give a range of lower limits on the fundamental Planck scale that could be obtained at LHC energies. In addition, we examine the effects of parton electric charge on black hole production using the trapped-surface approach of general relativity. Accounting for electric charge of the partons could reduce the black hole cross-section by one to four orders of magnitude at the LHC.
\end{abstract}

Keywords: black holes, extra dimensions, beyond Standard Model.

PACS numbers: 04.70.Bw, 04.50.+h, 12.60.-i, 04.70.-s

\section{Introduction}

Models of large $\sqrt{1 / 2 \mid 3}$ or warped $\frac{4 \mid 5}{4}$ extra dimensions allow the fundamental scale of gravity to be as low as the electroweak scale. For energies above the gravity scale, black holes can be produced in particle collisions. This opens up the possibility to produce black holes at the Large Hadron Collider (LHC). Once formed, the black hole will decay by emitting Hawking radiation $[6$ The final fate of the black hole is unknown since quantum gravity will become important as the black hole mass approaches the Planck scale. If black holes are produced at the LHC, detecting them will not only test general relativity and probe extra dimensions, but will also teach us about quantum gravity.

Early discussions of black hole production in colliders postulated a $\pi R_{\mathrm{S}}^{2}$ form for the cross-section, where $R_{\mathrm{S}}$ is the Schwarzschild radius of the black hole formed in the parton scattering process. $\frac{78 \mid 9}{}$ Calculations based on classical general relativity have had limited success in improving the cross-section estimates 1011 The effects of mass, spin, charge, colour, and finite size of the incoming particles are usually

${ }^{*}$ Also at TRIUMF, Vancouver, BC V6T 2A3, Canada. 
neglected. The effects of finite size have been examined $12[13$ and only recently have charge ${ }^{14}$ and spin 15 been discussed. Attempts have been made to account for angular momentum in a heuristic way by multiplying the simple expression for the cross-section by a form factor. $\frac{16[17|18| 19}{}$ Although these results are far from complete, they do indicated that the simple geometric cross-section is correct if multiplied by a formation factor of order unity 20

General relativistic calculations of the cross-section have usually been performed using the trapped-surface approach. The two incoming partons are modelled as Aichelburg-Sexl shock waves ${ }^{21}$ Spacetime is flat in all regions of space except at the shocks. The union of these shock waves defines a closed trapped surface. Black hole formation can be predicted by identifying a future trapped surface, with no need to calculate the gravitational field.

The trapped-surface approach was first applied to TeV-scale gravity calculations by Eardley and Giddings 10 in four dimensions. Their work was extended to the $(n+4)$-dimensional case numerically by Yoshino and Nambu 11 The numerical studies were improved by Yoshino and Rychkov 22 by analyzing the closed trapped surface on a future slice of spacetime. These general relativistic calculations have enabled lower limits to be obtained for the black hole mass produced by colliding particles in $\mathrm{TeV}$-scale gravity scenarios.

Since black holes are highly massive objects, the momentum fraction of the partons in the protons that form them must be high. Thus typically valence quarks will be involved in black hole formation. This means the most probable charge of the black hole in proton collisions will be $+4 / 3$. Since the gravitational field of each particle is determined by its energy-momentum tensor, charge should affect the black hole formation. First exploratory work by Yoshino and Mann $\frac{14}{14}$ obtained a condition on the electric charges of the colliding particles for a closed trapped surface to form. The results depend on the Standard Model brane thickness.

In this paper, we take trapped energy into account and give limits on the crosssection. We derive lower limits on the Planck scale based on estimates of the crosssection. 20 We use the Yoshino and Mann charge condition in its general form and build on their work by examining the effect of charge on black hole production at the LHC 23

\section{Classical Parton Cross-Section}

Black hole solutions in higher dimensions have a complicated dependence on both the gravitational field of the brane and the geometry of the extra dimensions. However, there are two useful approximations that may be made for a wide class of models. The brane on which the Standard Model particles live, is expected to have a tension given by roughly the Planck or string scale. For black holes with mass $M$ substantially heavier than the fundamental Planck scale in higher dimensions $M_{D}$, the brane's field should be negligible and the production process for black holes should be non-perturbative. We will assume that the only effect of the brane field 
is to bind the black hole to the brane, and that otherwise the black hole may be treated as an isolated object in the extra dimensions. If the geometrical scales of the extra dimensions $R$ (radii, curvature radii, variation scale of the warp factor) are all large compared to $1 / M_{D}$, there is a wide regime in which the geometry of the extra dimensions plays no essential role. We consider black holes with horizon radius $r_{\mathrm{h}}$ much smaller than the size of the extra dimensions, $1 / M_{D}<r_{\mathrm{h}} \ll R$. Under these assumptions, it is often a good approximation to consider the high-energy collision of the particles and the black hole formed to be in $(n+4)$-dimensional flat spacetime.

Since the black hole is not an ordinary particle of the Standard Model and its correct quantum theoretical treatment is unknown, it is treated as a quasi-stable state, which is produced and decays according to the semiclassical formalism of black hole physics. Using the above approximations, it has been argued that at high energies black hole production has a good classical description ${ }^{[9]}$ This leads to the naive estimate that the cross-section for black hole production is approximately given by the classical geometric cross-section $\hat{\sigma}=\pi R_{\mathrm{S}}^{2}$, where $R_{\mathrm{S}}$ is the $(n+4)$ dimensional Schwarzschild radius corresponding to the black hole mass. It depends on the fundamental Planck scale $M_{D}$ and the number of extra dimensions $n$. In the high-energy limit, the cross-section should depend on the impact parameter $b$ between the two partons, and a range of black hole masses will result for a given center of mass energy $\sqrt{\hat{s}}$. Since the cross-section is dominated geometrically by large impact parameters $b \lesssim R_{\mathrm{S}}$, the average black hole mass should be of the order of the center of mass energy, $\langle M\rangle \lesssim \sqrt{\hat{s}}$. It is often assumed that the black hole mass is given by $M=\sqrt{\hat{s}}$.

In studying the uncertainties in the classical parton cross-section, it is useful to examine a more general form of the cross-section

$$
\hat{\sigma}=F \pi r_{\mathrm{h}}^{2} \Theta\left(M-M_{\min }\right),
$$

where $F$ is a form factor (usually approximated as unity), $r_{\mathrm{h}}$ is a more general horizon that may depend on the angular momentum and charges of the black hole (usually taken to be the non-rotating non-charged Schwarzschild radius in flat $(n+$ 4)-dimensions), and $\Theta$ is a Heaviside step function that allows black hole production only above some threshold mass $M_{\text {min }}$ (often implicitly assumed). In addition, $M<$ $\sqrt{\hat{s}}$ needs to be considered to allow for the possibility of not all the available energy being trapped behind the horizon.

There exists a threshold for black hole production. In classical general relativity, two point-like particles in a head-on collision with zero impact parameter will always form a black hole, no matter how large or small their energy. At small energies, we expect this to be impossible due to the smearing of the wave function by the uncertainty relation. This then results in a necessary minimal energy to allow the required close approach. The threshold is of order $M_{D}$, though the exact value is unknown since quantum gravity effects should play an important role for the wave 
function of the colliding particles. For simplicity, it is usual to set this threshold equal to $M_{D}$.

In the high-energy limit, if the impact parameter is less than $r_{\mathrm{h}}$, a black hole with mass $M \sim \sqrt{\hat{s}}$ can be produced. To avoid quantum gravity effects and stay in the classical regime, we require $M \geq M_{\min }$, where $M_{\text {min }}$ should be a few times larger than $M_{D}$, although it is often taken as $M_{D}$. A reasonable criterion for $M_{\text {min }}$ is given by the requirement of large entropy 24 In the following, we will find it useful to define the dimensionless parameter

$$
x_{\min }=\frac{M_{\min }}{M_{D}},
$$

and require $x_{\min } \gg 1$.

Throughout this paper, we use the Particle Data Group (PDG) ${ }^{25}$ definition of the Planck scale

$$
M_{D}^{n+2}=\frac{1}{8 \pi G_{\mathrm{N}}} \frac{1}{R^{n}},
$$

where $G_{\mathrm{N}}$ is Newton's constant in four dimensions.

\section{Particle Cross-Section}

Only a fraction of the total center of mass energy $\sqrt{s}$ in a proton-proton collision is available in the parton-parton scattering process. We define $s x_{\mathrm{a}} x_{\mathrm{b}} \equiv s \tau \equiv \hat{s}$, where $x_{\mathrm{a}}$ and $x_{\mathrm{b}}$ are the fractional energies of the two partons relative to the proton energies. The full particle-level cross-section $\sigma$ is obtained from the parton-level cross-section $\hat{\sigma}$ by using

$$
\sigma_{p p \rightarrow \mathrm{BH}+\mathrm{X}}(s)=\sum_{\mathrm{a}, \mathrm{b}} \int_{\frac{M^{2}}{s}}^{1} d \tau \int_{\tau}^{1} \frac{d x}{x} f_{\mathrm{a}}\left(\frac{\tau}{x}\right) f_{\mathrm{b}}(x) \hat{\sigma}_{\mathrm{ab} \rightarrow \mathrm{BH}}\left(\hat{s}=M^{2}\right),
$$

where $f_{\mathrm{a}}$ and $f_{\mathrm{b}}$ are parton distribution functions (PDFs) for the proton. The sum is over all possible quark and gluon pairings. Throughout this paper, we use the CTEQ6L1 (leading order with leading order $\alpha_{s}$ ) parton distributions functions 26 within the Les Houches Accord PDF framework We have taken $Q=R_{\mathrm{S}}^{-1}$ for the QCD scale.

Since $\hat{s}=M^{2}$, we can make a changing of variable from $\tau$ to $M$ to obtain the differential cross-section in terms of parton luminosity (or parton flux)

$$
\frac{d \sigma_{p p \rightarrow \mathrm{BH}+\mathrm{X}}}{d M}=\frac{d L}{d M} \hat{\sigma}_{\mathrm{ab} \rightarrow \mathrm{BH}}, \text { where } \frac{d L}{d M}=\frac{2 M}{s} \sum_{\mathrm{a}, \mathrm{b}} \int_{M^{2} / s}^{1} \frac{d x}{x} f_{\mathrm{a}}\left(\frac{\tau}{x}\right) f_{\mathrm{b}}(x) .
$$

${ }^{a}$ LHAPDF the Les Houches Accord PDF Interface, Version 5.2.2, maintained by M. Whalley; http://hepforge.cedar.ac.uk/lhapdf/ 
The differential cross-section thus factorizes for the case of $\hat{s}=M^{2}$. It can be written as the product of the parton cross-section time a luminosity function. The parton cross-section is independent of the parton types and depends only on the black hole mass, Planck scale, and number of extra dimensions. The parton luminosity function contains all the information about the partons. Beside a dependence on black hole mass, it is independent of the characteristics of the higher-dimensional space, i.e. the Planck scale and number of extra dimensions. The particle-level crosssection does not truly factorize if the horizon radius is used as the QCD scale in the parton density functions for the proton.

\section{Trapped Surfaces and Trapped Energy}

Classical general relativistic calculations indicate that the mass of a black hole formed in a head-on collision is somewhat less than the total center of mass energy; the scattering is not completely inelastic. Thus Eq. (1) should be modified by replacing the black hole mass by a fraction of the available center of mass energy, leading to a reduction in the cross-section.

To improve the naive picture of colliding point particles, we need to consider the grazing collision of particles in $(n+4)$-dimensional Einstein gravity and investigate the formation of trapped surfaces. A common approach is to treat the creation of the horizon as a collision of two shock fronts in Aichelburg-Sexl geometry. ${ }^{21}$ The Aichelburg-Sexl metric is obtained by boosting the Schwarzschild metric to form two colliding shock fronts. Due to the high velocity of the moving particles, spacetime before and after the shocks is almost flat and the geometry can be examined for the occurrence of trapped surfaces, which depend on the impact parameter.

Eardley and Giddings 10 developed a method for finding the trapped surfaces for this system. For a nonzero impact parameter, they were able to solve the problem analytically for the $n=0$ case. They obtained limits on the final mass of the black hole formed and found a range from $M>0.71 \sqrt{\hat{s}}$ for $b=0$ to $M>0.45 \sqrt{\hat{s}}$ for $b=$ $b_{\max }$. This can be compared with a perturbative analysis that gave $M \approx 0.8 \sqrt{\hat{s}}$. 27 For higher dimensions, they solved the $b=0$ case to obtain lower bounds on the final black hole mass of $M>0.71 \sqrt{\hat{s}}$ to $0.589 \sqrt{\hat{s}}$ for $n=0$ to 7 .

Unfortunately the Eardley and Giddings results are not general enough to be useful for nonzero impact parameters and higher dimensions, but they indicate that a significant amount of the initial energy may not be trapped behind the horizon. Understanding the case of a nonzero impact parameter in higher dimensions is crucial to improving the cross-section estimates. The analytic techniques used to study head-on collisions in general relativity are not applicable to collisions at nonzero impact parameter. Thus the claim that a black hole will be produced when $b<r_{\mathrm{h}}$ can only be expected to be true up to a numerical factor.

Yoshino and Nambu ${ }^{11}$ solved this problem numerically for $n>0$ and obtained the maximal impact parameter $b_{\max }$. In their analysis, the trapped surface was constructed on the union of the two incoming shocks. However, this slice of spacetime is 
not optimal in the sense that there exists other slices located in the future. Yoshino and Rychkov 22 improved the analysis by using a future slice. They found that the lower bound on the black hole mass formed is never more than $71 \%$ of the available energy. The fraction of energy available decreases with impact parameter and the number of extra dimensions, from 0.71 to 0.46 for $n=0$ and from 0.59 to 0 for $n=7$. The mean lower bound on the trapped energies are about 0.6 and 0.27 for $n=0$ and 7 respectively.

The following describes one approach to taking estimates of the non-trapped energy into account, and applying a minimum black hole mass cutoff to final results. We use the Yoshino and Rychkov 22 data to obtain lower bounds on the black hole cross-section.

\section{Inelastic Particle Cross-Section}

Previous calculations of the cross-section for producing a black hole have neglected energy loss, and assumed that the mass of the created black hole was identical to the incoming parton center of mass energy. However, recent work $\frac{11 / 22}{2}$ shows the energy loss to gravitational radiation is not negligible, and in fact is large for large number of extra dimensions and for large impact parameters.

The trapped mass $M$ is given by (using the notation of Anchordoqui et al. ${ }^{28}$ )

$$
M(z)=y(z) \sqrt{\hat{s}},
$$

where the inelasticity $y$ is a function of $z \equiv b / b_{\max }$. This complicates the parton model calculations, since the production of a black hole of mass $M$ is lower than $\sqrt{\hat{s}}$ by $M / y(z)$, thus requiring the lower cutoff on the parton momentum fraction to be a function of the impact parameter. We can no longer use the factorized version of the particle-level cross-section given by Eq. (5).

Following Anchordoqui et al. ${ }^{24 \mid 28}$, we take the proton-proton cross-section as the impact parameter-weighted average over parton cross-sections, with the lower parton fractional momentum cutoff determined by $M_{\text {min }}$. This gives a lower bound $\left(x_{\min } M_{D}\right)^{2} /\left(y^{2} s\right)$ on the parton momentum fraction $x$. With this in mind, the $p p \rightarrow \mathrm{BH}+\mathrm{X}$ cross-section becomes

$$
\sigma_{p p \rightarrow \mathrm{BH}+\mathrm{X}}\left(s, x_{\min }\right) \geq \int_{0}^{1} 2 z d z \sum_{\mathrm{a}, \mathrm{b}} \int_{\frac{\left(x_{\min M} M_{D}\right)^{2}}{y^{2} s}}^{1} d \tau \int_{\tau}^{1} \frac{d x}{x} f_{\mathrm{a}}\left(\frac{\tau}{x}\right) f_{\mathrm{b}}(x) \hat{\sigma}_{\mathrm{ab} \rightarrow \mathrm{BH}}(\tau s) .
$$

Since the amount of trapped energy is a lower bound, the resulting cross-section is a lower bound.

Taking $x_{\min }=1$, we obtain the families of cross-section curves shown in Figs. 1 and 2. The solid curves are for the classical cross-section calculated using Eqs. (1) and (5) with the form factors of Ref. 22. We will henceforth refer to these curves as the classical cross-section. The dashed lower curves are given by Eqs. (11) and 

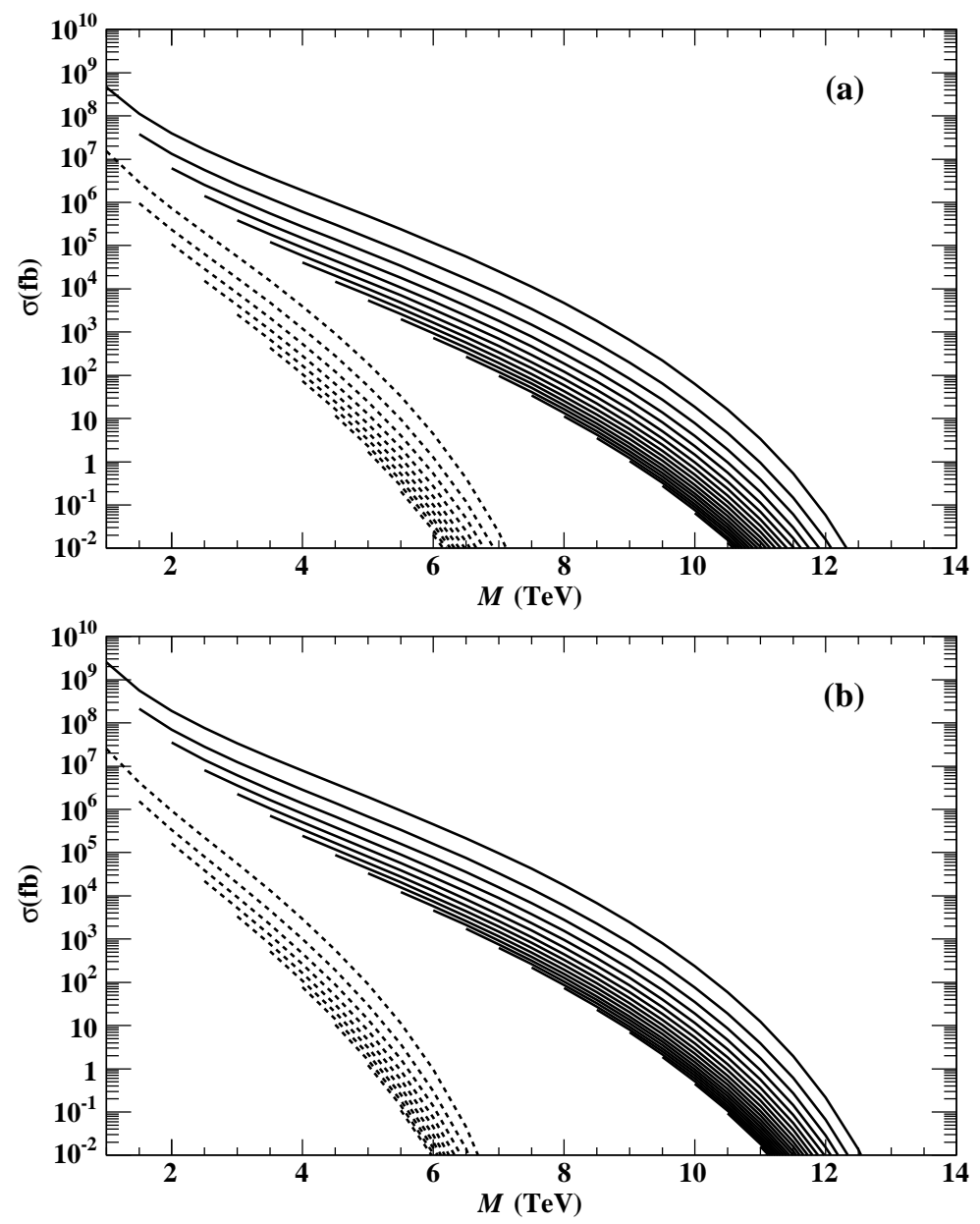

Fig. 1. Cross-section versus black hole mass. Solid curves classical cross-section and dashed curves trapped-surface cross-section. Curves of same type for different Planck scales, $0.5 \mathrm{TeV}$ top curves decreasing with increasing Planck scale in steps of $0.5 \mathrm{TeV}$. (a) $n=3$ and (b) $n=7$. Ref. 20

(17) with the form factors of Ref. 22. We will henceforth refer to these curves as the trapped-surface cross-section. In Figs. 1(a) and 1(b) the different curves of a given type are for different Planck scales, starting from $0.5 \mathrm{TeV}$ for the top curve and decreasing with increasing Planck scale in steps of $0.5 \mathrm{TeV}$. Figure 1 (a) is for $n=3$, while Fig. 1(b) is for $n=7$. In Figs. 2(a) and 2(b) the different curves of a given type are for different numbers of extra dimensions, starting from $n=2$ for the top curve and ending at $n=7$ for the bottom curve. Figure 2(a) is for a Planck scale of $1 \mathrm{TeV}$, while Fig. 2(b) is for a Planck scale of $5 \mathrm{TeV}$.

The effect of non-trapped energy on the cross-section is large because the LHC energy is close to the threshold for black hole production and lost energy limits 

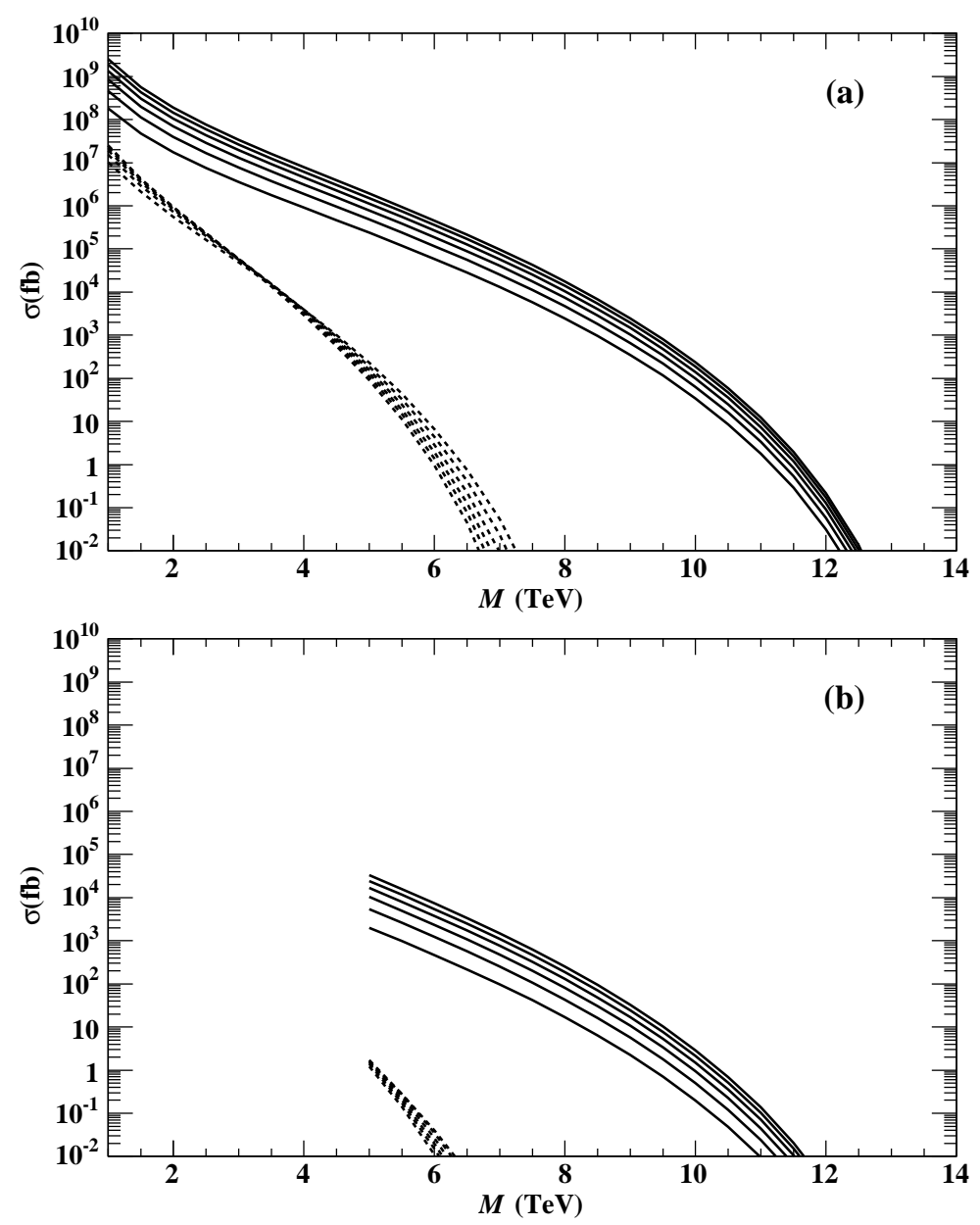

Fig. 2. Cross-section versus black hole mass. Solid curves classical cross-section and dashed curves trapped-surface cross-section. Curves of same type for different number of extra dimensions, top curves $n=2$ and bottom curves $n=7$. (a) $M_{D}=1 \mathrm{TeV}$ and (b) $M_{D}=5 \mathrm{TeV}$. Ref. 20]

the availability energy for the black hole. The cross-section curves show that there is less dependence on $n$ than $M_{D}$. This is because the $n$ dependence of the form factor tends to cancel the $n$ dependence of the horizon radius. 29 It is reasonable to consider the classical cross-section with form factors greater than unity as loose upper bounds on the black hole cross-section, which may increase by a factor of a few as the formation factors increase. We thus take the point of view that the black hole cross-section lies between the classical and trapped-surface cross-sections. The difference can be several orders of magnitude. The trapped-surface cross-sections cut off at a mass above the trapped-energy bounds. Applying a cutoff $x_{\min }>1$ will further restrict the range of the trapped-surface cross-sections, as well as the 
classical cross-sections.

\section{Lower Limits on the Planck Scale}

The cross-sections in the previous section can be used to predict the discovery limits for a given luminosity and be used, in principle, to extract the Planck scale and number of extra dimensions. In the event of no detectable black hole signal, the cross-sections can also be used to set limits on the Planck scale and number of extra dimensions.

We consider the scenario in which no black hole signal has been observed after the accumulation of an integrated luminosity of $300 \mathrm{fb}^{-1}$ at $\sqrt{s}=14 \mathrm{TeV}$. Rather than study the different decay phases of the black hole and estimate the detector's capabilities for measuring them, we assume a perfect detector. This will give the most optimistic limits possible. Assuming a perfect experiment, the $95 \%$ confidencelevel upper limit on the cross-section is $10^{-2} \mathrm{fb}$. Using this value of the crosssection, we have extracted lower limits on the Planck scale $M_{D}$ as a function of cutoff parameter $x_{\min }$ for different values of the number of extra dimensions $n$. The results are shown in Fig. 3 for $n=2$ to 7 . The solid curves were obtained from the classical cross-sections. The dashed curves were obtained from the trappedsurface cross-section bounds. The dotted curves are a result of the mass cutoff in the trapped-surface cross-sections. The dotted curves can be consider as the infinite luminosity case of the trapped-surface predictions. The small spread in the different curves of a given type is due to the different number of extra dimensions.

We can use Fig. 3 to get a feel for how the different cross-section models affect the range of Planck-scale limits. For $x_{\min }=5$, a lower limit of $M_{D}>2.4 \mathrm{TeV}$ is obtained for the classical case and $M_{D}>1.4 \mathrm{TeV}$ for the trapped-surface case. The trapped-surface limit can be improved to $M_{D}>1.7 \mathrm{TeV}$ with infinite luminosity. Relaxing the cutoff criteria used to avoid quantum gravity effects to $x_{\min }=3$ gives a lower limit of $M_{D}>3.8 \mathrm{TeV}$ for the classical case and $M_{D}>2.2 \mathrm{TeV}$ for the trapped-surface case. The trapped-surface limit can be improved to $M_{D}>2.8 \mathrm{TeV}$ with infinite luminosity. There appears to be very little sensitivity to the limits on the Planck scale due to the number of extra dimensions: less than a $3 \%$ effect.

\section{7. $(n+4)$-Dimensional Reissener-Nordström Spacetime}

Yoshino and Mann ${ }^{14}$ have studied the effects particle charge on black hole formation by replacing the usual flat $(n+4)$-dimensional Schwarzschild metric by the flat $(n+4)$-dimensional Ressener-Nordström metric, and searching for closed trapped surfaces on the future lightcone. They followed the usual Aichelburg-Sex 21 prescription by boosting a pair of metrics representing the gravitational fields of two point particles of mass $m$ and charge $q$ in $(n+4)$ dimensions in a head-on collision to the likelike limit $(\gamma \rightarrow \infty)$. In the process, the total energy $E=\gamma m$ and the quantity $p_{e}^{2}=\gamma q^{2}$ are keep fixed. The charge dependence of the equivalent Achelburg-Sexl metric is entirely contained in an additional term that depends on 


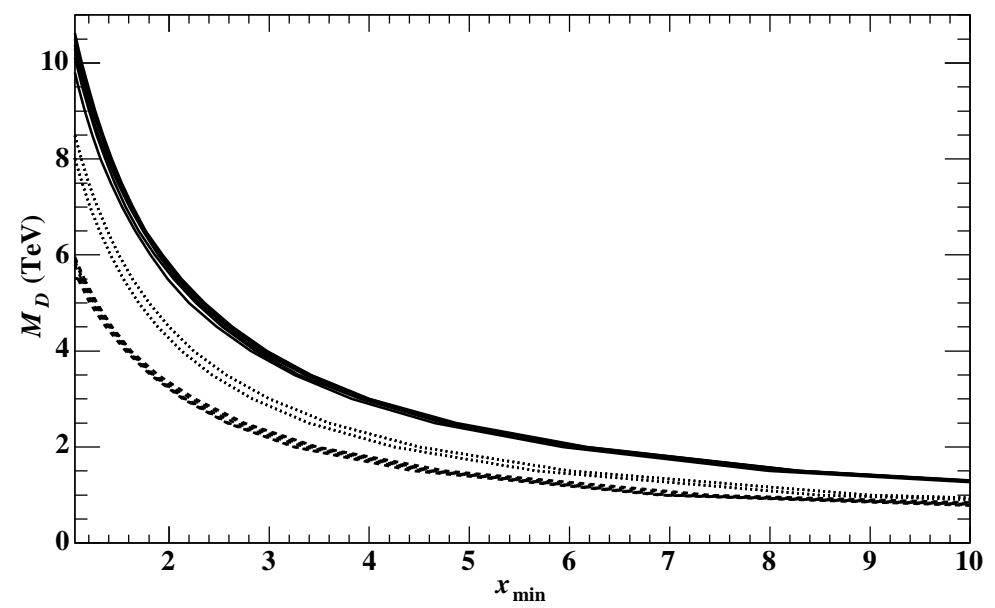

Fig. 3. Lower limits on Planck scale as function of cutoff parameter. Solid curves classical case. Dashed curves trapped-surface case. Dotted curves result of mass cutoff in trapped-surface case. Spread in curves of same type due to different $n$. Ref. 20.

$$
a=\frac{2 \pi\left(4 \pi G_{D} p_{e}^{2}\right)}{n+1} \frac{(2 n+3) ! !}{(2 n+4) ! !} .
$$

We refer to $a$ as a generalized charge.

The apparent horizon is given by the union of two surfaces that are a monotonically increasing function $r$. When the two surfaces cross at the instance of collision $r=r_{\text {min }}$. Imposing this boundary condition on the differential equation for the apparent horizon gives 14

$$
x^{4}=\left(x-a_{1}\right)\left(x-a_{2}\right),
$$

where $x \equiv r_{\min }^{n+1}$, and $a_{1}$ and $a_{2}$ are given by Eq. (8) with the corresponding values of $p_{e}^{(1)}$ and $p_{e}^{(2)}$. Equation (9) determines the value of $r_{\min }$. The apparent horizon exists if, and only if, there is a solution to Eq. (9) with $x>a_{1}$ and $x>a_{2}$.

Figure 4 shows the region for apparent horizon formation in the $\left(a_{1}, a_{2}\right)$-plane. We see that both $a_{1}$ and $a_{2}$ must be sufficiently small for an apparent horizon to form. For two particles of equal charge, $a_{1}=a_{2}=1 / 4$ is a solution of Eq. (9). For one charged particle and one neutral particle, $a_{1}=2 /(3 \sqrt{3})$ and $a_{2}=0$ is also a solution of Eq. (9).

We can understand the requirement on $a_{1}$ and $a_{2}$ physically as follows. Since $a_{1}$ and $a_{2}$ are proportional to $\left(p_{e}^{(1)}\right)^{2}$ and $\left(p_{e}^{(2)}\right)^{2}$, the condition derived in Eq. (9) does not depend on the sign of the charge of either particle. This is because the gravitational field due to each charge is generated by an electromagnetic energymomentum tensor $T_{\mu \nu}^{(\mathrm{em})}$ that depends on the square of the charge. The gravitational 


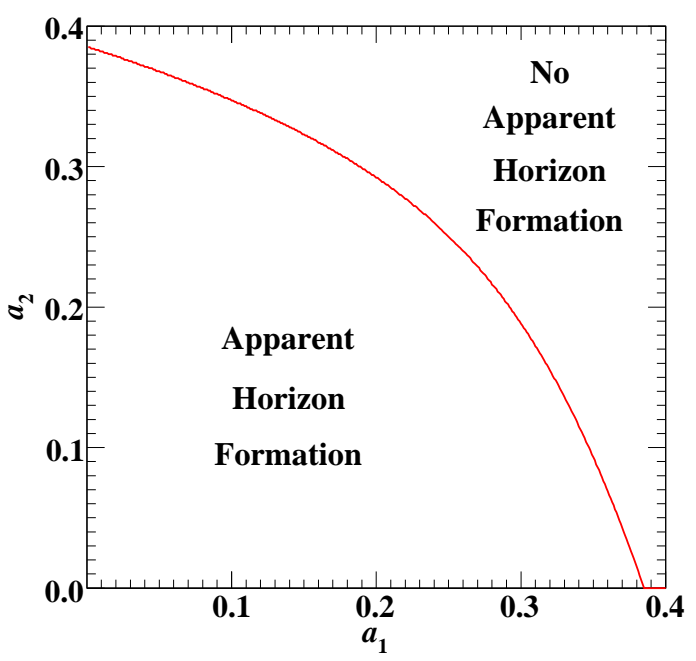

Fig. 4. Region of apparent horizon formation in $\left(a_{1}, a_{2}\right)$-plane. Ref. 23

field induced by $T_{\mu \nu}^{(\mathrm{em})}$ of the incoming particles is repulsive, and its affect becomes dominant around the center. As the value of $a$ increases, the repulsive region becomes larger, preventing formation of the apparent horizon. The critical value of $a$ for apparent horizon formation occurs when the repulsive gravitational force due to the electric field becomes equivalent to the self-attractive force due to the energy of the system.

The approach for handling the confinement of the electric field to the Standard Model three-brane is far from clear. So far, we have ignored this effect by using the $(n+4)$-dimensional Einstein-Maxwell theory. We develop the relationship between the electric charge in four dimensions $q_{4}$ and the charge in higher-dimensional Maxwell theory $q$. For two particles in $(n+4)$ dimensions with the same charge at rest, for example, the force between them is $F=q^{2} / r^{n+2}$. If the gauge fields are confined to the Standard Model brane, the only characteristic length scale is the width of the brane, which should be of the order of the Planck length. We introduce the constant $C_{\text {brane }}: 1 / M_{D} \rightarrow C_{\text {brane }} / M_{D}$, where $C_{\text {brane }}$ is a dimensionless quantity of order unity. For sufficiently large $r$,

$$
q^{2}=q_{4}^{2}\left(\frac{C_{\text {brane }}}{M_{D}}\right)^{n}
$$

The brane thickness is a measure of how confined the Standard Model electric charge is to the brane. If the brane is thick, the Maxwell theory would be higher dimensional in the neighbourhood of the particle. We let $q_{4}^{2}=C_{q}^{2} \alpha$, where $C_{q}$ is the charge in units of elementary charge $e(-1 / 3$ or $+2 / 3$ for quarks and 0 for gluons) and $\alpha$ is the fine structure constant. Our treatment of the electric charge has not 
fully taken into account the effects of confinement of the electric field on the brane. We have also ignored the brane tension and the structure of the extra dimensions.

From the above, the general charge becomes

$$
\frac{a}{r_{0}^{2(n+1)}}=C_{q}^{2} \alpha\left(\frac{M_{D}}{m}\right)\left(\frac{M_{D}}{E}\right) \pi \frac{\Omega_{n+1}^{2}}{n+1} \frac{(2 n+3) ! !}{(2 n+4) ! !}\left(\frac{C_{\text {brane }}}{2 \pi}\right)^{n}
$$

where $r_{0}=\left(8 \pi G_{D} E / \Omega_{n+1}\right)^{\frac{1}{n+1}}$.

Choosing values for $C_{q}$ and $m$, we can use Eq. (11) to study the condition for apparent horizon formation as a function of $n, M_{D}$, and $C_{\text {brane }}$. An apparent horizon will not occur at the instance of collision if the brane is thick or if the spacetime dimensionality is low. Charge effects will not be significant at high energies.

\section{Effect of Charged Partons on the Cross-Section}

We work with parton luminosity, which is independent of $n$ and $M_{D}$. Only the condition on which quarks to include in the sum of Eq. (5) depends on $n$ and $M_{D}$. Thus the upper and lower bounds on the parton luminosity do not change for different parameters. To a good approximation, we can ignore the contribution from the sea quarks at high black hole masses. The gluon contribution is the lower bound on the luminosity function when the charged quarks do not contribute to the cross-section. We take the running of the coupling constant into account; we choose $\alpha$ equal to $1 / 124$ in the following calculations. Because of the large momentum transfer in black hole production, we use current quark masses. Quark masses of $m_{\mathrm{d}}=8 \mathrm{MeV}$ and $m_{\mathrm{u}}=4 \mathrm{MeV}$ are chosen for the valence quarks in the proton. To study Eq. (5), we must first calculate Eq. (11), and then determine if the condition in Fig. 4 is satisfied. If it is, the parton pair is included in the sum in Eq. (5).

Figure 5 shows the parton luminosity for different brane thicknesses for seven extra dimensions and a Planck scale of $1 \mathrm{TeV}$. The top curve is the case when all the partons contribute to the cross-section, while the lower curve is the case when only the neutral gluons contribute to the cross-section. The contributions of the different quarks in the intermediate region depends on $M, n, M_{D}$, and $C_{\text {brane }}$. The thresholds for different quarks to contribute occur as a function of $M$ for fixed $n, M_{D}$, and $C_{\text {brane. }}$ The location of the thresholds may or may not occur in the mass region of our plot. From Fig. [5, we see that charge can affect any black hole mass and the effect is very sensitive to the brane thickness. The decrease in parton luminosity, and thus cross-section, can range from about one to four orders of magnitude over a black hole mass range of 1 to $10 \mathrm{TeV}$ due to charge effects. The cross-section is nontrivial only over a range of brane thicknesses from 1.1 to 2.2. Plots with different number of dimensions are similar to Fig. 5 they are always bounded above and below by the same values, but for different values of the brane thickness.

For each number of dimensions, we determine the maximum brane thickness for all partons to be included in the parton luminosity and the minimum brane thickness 


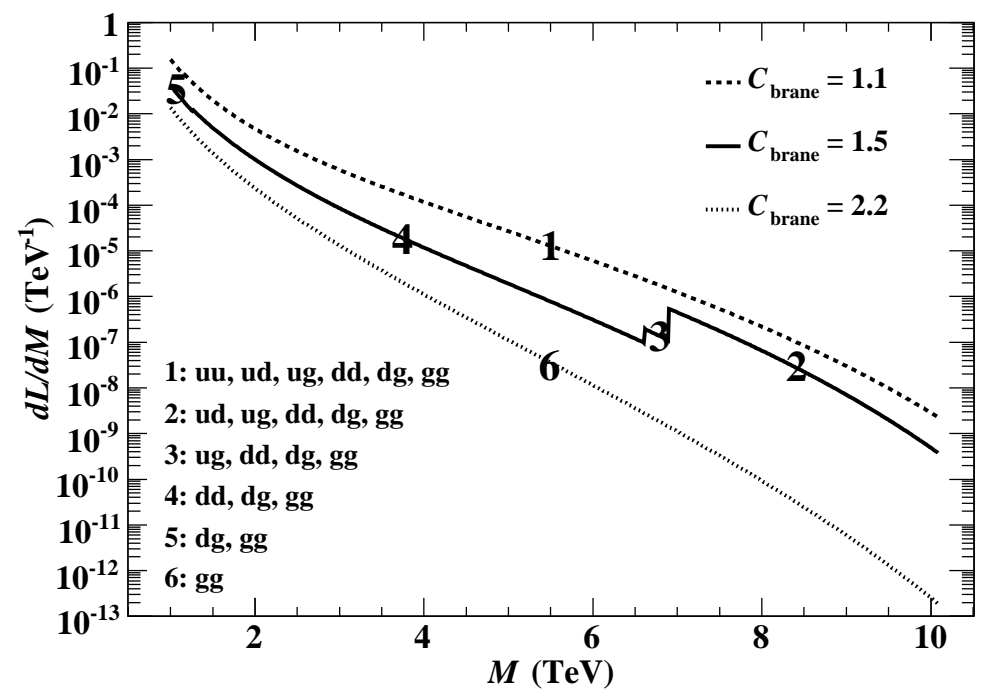

Fig. 5. Parton luminosity function versus black hole mass with charge condition applied for different brane thicknesses for $n=7$ and $M_{D}=1 \mathrm{TeV}$. Numbers on plot show different parton contributions to parton luminosity function. Ref. 23

for only gluons to be included in the parton luminosity. The results are shown in Fig. 6. For a thin brane, the cross-section is not affected for high dimensions. For a thick brane, the cross-section is reduced for most number of dimensions. For a Planck scale of $1 \mathrm{TeV}$ and a brane thickness of $1 \mathrm{TeV}^{-1}$, the cross-section is minimal for $n \lesssim 4$, not affected for $n \gtrsim 7$, and has a range of values in the region $5 \lesssim n \lesssim 6$.

\section{Discussion}

The limits on $M_{D}$ presented here are compatible with the discovery limits that have been determined in previous work. $24|28| 30|31| 32 \mid 33$ Our limits might appear different due to the stringent requirements on $x_{\min }$ and the different definition of $M_{D}$. The large difference between the classical and trapped-surface cross-sections does not translate into a large difference in the limits on $M_{D}$ because both crosssections fall rapidly at low values of the cross-section. If one is willing to relax the requirement on $x_{\min }$ and risk entering the quantum-gravity regime, than the differences between the two models becomes significant. This difference presumably still holds when the uncertainties in the black hole decay and experimental effects are taken into account.

The trapped-energy approach only gives a lower bound on the final mass of the black hole. In order to clarify the final mass, different methods such as the direct study of gravitational wave emission are necessary 34 The problem is extremely difficult because of the nonlinearity of Einstein's equations, and because the highenergy collision of the two particles producing a black hole requires inclusion of 


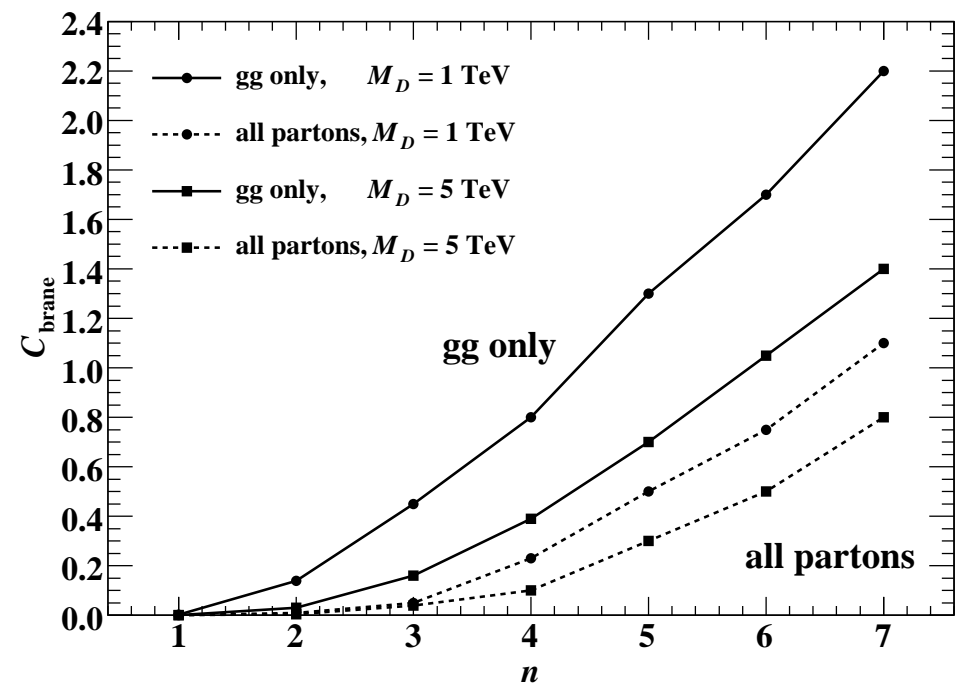

Fig. 6. Brane thickness versus number of dimensions for which all quarks (dashed lines) and no quarks (solid lines) contribute to cross-section. Circles are for $M_{D}=1 \mathrm{TeV}$ and squares are for $M_{D}=5 \mathrm{TeV}$. No quarks contribute to cross-section in region above solid curves. Cross-section is not affect by charge condition below region of dashed curves. Some quarks contribute to crosssection in region between different curve types. Ref. 23

nonlinear effects.

Taking the boosted Reissner-Nordström metric as a reasonable description of ultarelativistic quarks, we have shown that charge effects will significantly decrease the rate of black hole formation at the LHC, if the brane is somewhat thick or if the number of extra dimensions $n$ is not too large. The charge effects can be quite large because the electromagnetic energy-momentum tensor is proportional to $p_{e}^{2} \sim \gamma \alpha$ and the Lorentz factor $\gamma$ is much larger than $1 / \alpha$ for ultrarelativistic quarks.

There are many uncertainties in our understanding of black hole production in higher dimensional $\mathrm{TeV}$-scale gravity. Reliable predictions of the cross-section are not yet available. We have explored some options for filling in the gaps in our understanding. In this way, we hope to be better prepared to confront the possibility of black hole production at the LHC.

\section{References}

1. N. Arkani-Hamed, S. Dimopoulos and G. Dvali, Phys. Lett. B 429, 263 (1998).

2. I. Antoniadis, N. Arkani-Hamed, S. Dimopoulos and G. Dvali, Phys. Lett. B 436, 257 (1998).

3. N. Arkani-Hamed, S. Dimopoulos and G. Dvali, Phys. Rev. D 59, 086004 (1999).

4. L. Randall and R. Sundrum, Phys. Rev. Lett. 83, 3370 (1999).

5. L. Randall and R. Sundrum, Phys. Rev. Lett. 83, 4690 (1999).

6. S. W. Hawking, Commun. math. Phys. 43, 199 (1975). 
7. T. Banks and W. Fischler, A model for high energy scattering in quantum gravity, hep-th/9906038

8. S. Dimopoulos and G. Landsberg, Phys. Rev. Lett. 87, 161602 (2001).

9. S. B. Giddings and S. Thomas, Phys. Rev. D 65, 056010 (2002).

10. D. M. Eardley and S. B. Giddings, Phys. Rev. D 66, 044011 (2002).

11. H. Yoshino and Y. Nambu, Phys. Rev. D 67, 024009 (2003).

12. E. Kohlprath and G. Veneziano, J. High Energy Phys. 0206, 057 (2002).

13. S. B. Giddings and V. S. Rychkov, Phys. Rev. D 70, 104026 (2004).

14. H. Yoshino and R. B. Mann, Phys. Rev. D 74, 044003 (2006).

15. H. Yoshino, A. Zeinikov and V. P. Frolov, Apparent horizon formation in the head-on collision of gyratons, gr-qc/0703127.

16. S. C. Park and H. S. Song, J. Korean Phys. Soc. 43, 30 (2001).

17. A. V. Kotwal and C. Hays, Phys. Rev. D 66, 116005 (2002).

18. L. A. Anchordoqui, J. L. Feng, H. Goldberg and A. D. Shapere, Phys. Rev. D 65, 124027 (2002).

19. D. Ida, K. Oda and S. C. Park, Phys. Rev. D 67, 064025 (2003).

20. D. M. Gingrich, Int. J. Mod. Phys. A 21, 6653 (2006).

21. P. C. Aichelburg and R. U. Sexl, Gen. Relativ. Gravit. 2, 303 (1971).

22. H. Yoshino and V. S. Rychkov, Phys. Rev. D 71, 104028 (2005).

23. D. M. Gingrich, J. High Energy Phys. 0702, 098 (2007).

24. L. A. Anchordoqui, J. L. Feng, H. Goldberg and A. D. Shapere, Phys. Lett. B 594, 363 (2004).

25. Particle Data Group (W.-M. Yao et al.), J. Phys. G 33, 1 (2006).

26. J. Pumplin, D. R. Stump, J. Huston, H. L. Lai, P. Nadolsky and W. K. Tung, J. High Energy Phys. 0207, 012 (2002).

27. P. D. D'Eath, Class. Quantum Grav. 10, S207 (1993).

28. L. A. Anchordoqui, J. L. Feng, H. Goldberg and A. D. Shapere, Phys. Rev. D 68, 104025 (2003).

29. B. Webber, Black holes at accelerators, eConf C0507252, T030 (2005), hep-ph/0511128.

30. G. F. Giudice, R. Rattazzi and J. D. Wells, Nucl. Phys. B 630, 293 (2002).

31. E.-J. Ahn, M. Cavaglià and A. V. Olinto, Astropart. Phys. 22, 377 (2005).

32. J. Tanaka, T. Yamamura, S. Asai and J. Kanzaki, Eur. Phys. J. C 41(s02) 19 (2005).

33. C. M. Harris, M. J. Palmer, M. A. Parker, P. Richardson, A. Sabetfakhri and B. R. Webber, J. High Energy Phys. 0505, 053 (2005).

34. V. Cardoso, E. Berti and M. Cavaglià, Classical Quantum Gravity 22, L61 (2005). 